\title{
High Frequency Cutoff and Change of Radio Emission Mechanism in Pulsars
}

\author{
V. M. Kontorovich ${ }^{1,2}$ A. B. Flanchik ${ }^{1}$
}

\begin{abstract}
Pulsars are the fast rotating neutron stars with strong magnetic field, that emit over a wide frequency range. In spite of the efforts during 40 years after the discovery of pulsars, the mechanism of their radio emission remains to be unknown so far. We propose a new approach to solving this problem for a subset of pulsars with a high-frequency cutoff of the spectrum from the Pushchino catalogue (the "Pushchino" sample). We provide a theoretical explanation of the observed dependence of the high-frequency cutoff from the pulsar period. The dependence of the cutoff position from the magnetic field is predicted. This explanation is based on a new mechanism for electron radio emission in pulsars. Namely, radiation occurs in the inner (polar) gap, when electrons are accelerated in the electric field that is increasing from zero level at the star surface. In this case acceleration of electrons passes through a maximum and goes to zero when the electron velocity approaches the speed of light. All the radiated power is located within the radio frequency band. The averaging of intensity radiation over the polar cap, with some natural assumptions of the coherence of the radiation, leads to the observed spectra. It also leads to an acceptable estimate of the power of radio emission.
\end{abstract}

Keywords pulsars: radio emission, spectra

\section{Introduction}

Pulsars are magnetized neutron stars that have a magnetosphere filled with an electron-positron plasma of

\section{M. Kontorovich}

A. B. Flanchik

${ }^{1}$ Institute of Radio Astronomy of National Academy of Sciences of Ukraine, 4 Chervonopraporna Str., Kharkov 61002, Ukraine.

${ }^{2}$ Karazin Kharkov National University, 4 Svobody Sq., Kharkov 61077, Ukraine. about the GJ density (Smith 1977; Manchester \& Tavlor 1977; Beskin, et al. 1993). New discoveries of double pulsar system (Lane, et al. 2004) and intermittent pulsars (Kramer, et al. 2006; Lorimer. et al. 2012; Camilo, et al. 2012) give the direct observational support to that idea. It is thought that this plasma in the region of open magnetic field lines over the magnetic polar cap is generated by particles (through gamma quanta production) accelerating in a gap under the magnetosphere (Sturrock 1971; Ruderman \& Sutherland 1975; Arons 1981; Beskin 2010). The acceleration of electrons occurs in the gap in the electric field that is longitudinal with respect to the magnetic field and induced by the rotation of the magnetized star. Directed coherent electromagnetic radiation of relativistic particles from the region of open lines creates the beacon effect that results in the pulses observed (the most popular explanation).

In explanation of radio emission of pulsars (see reviews Malov 2004; Manchester 2009) and addition references in (Malov \& Machabeli 2009; Kontorovich 2009; Beskin \& Philippov 2012)) the instabilities of plasma flow, beam instabilities and similar effects in the magnetospheric plasma 11 have been discussed. Apparently, various mechanisms of radio emission are actually realized and may in certain circumstances succeed each other.

We show in this paper that for the observed pulsar radio emission a coherent radiation produced in a polar gap may be responsible, at least for pulsars of the

\footnotetext{
${ }^{1}$ Note apart the plasma-beam (see as example Usov 1987; Kazbegi, et al. 1992)), also the cyclotron, drift, modulation instabilities, Zakharov's wave collapse and magnetic reconnection for GP, low-frequency "tails" of the synchrotron, Cherenkov, Doppler and curvature radiation in the relativistic electronpositron plasma.
} 
Pushchino sample 2 with cutoff in the radio spectrum. The relationships we have received we will also use for the pulsar in the Crab Nebula, given its peculiarities. The radiation has been emitted with the acceleration of electrons in the gap. It is quite essential that the accelerating longitudinal electric field in the gap slowly increases from zero at rising off the star surface. This mechanism has never been discussed earlier for pulsars.

In the well-known Ruderman-Sutherland model the strong electric field, non vanishing at the star surface, accelerates electrons so quickly that their radiation due to acceleration in the gap fully comes to the hard energy (X-ray) region with no radio emission. Only in an electric field slow rising from zero level on the star surface the radiation of accelerating electrons comes to the radio band.

Resulting radiation is limited by a cutoff frequency found in this study. It coincides with the high-frequency cutoff (Malofeev \& Malov 1980; Malov 2004) in the pulsar spectra of the Pushchino sample. Such frequency limitation is due to the fact that the electron acceleration in the electric field, vanishing on the star surface, passes through a maximum and decreases as the electron velocity approaches the relativistic limit.

There is the all-new specification of our point of view and the main result of this work.

${ }^{2}$ The sample is based on Pushchino catalogue Malofeev 1996, 1999). Catalogue gathers simultaneous and compiled radio spectra for 340 pulsars (on more than three frequencies) with more than 120 references on measurements on different instruments including Bonn 100m and Ukrainian decameter radio telescopes. The correlation between frequency maximum, cutoff frequency and period have been found for some part of the spectra (Malofeev \& Malov 1980). We don't touch here the more high frequency values in catalogue (Maron 2000) with new results that ask for separate investigation, see also discussion in (Sieber 2002).

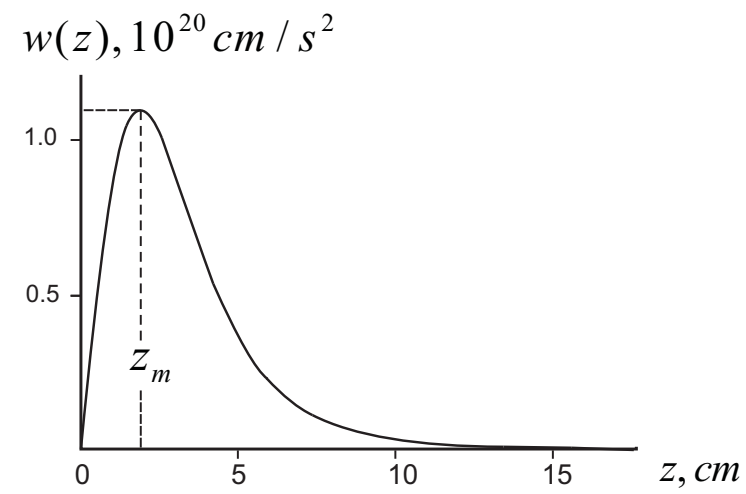

Fig. 1 The dependence of the acceleration on the altitude $z$ on the star surface

\section{Acceleration of electrons in the speed up process}

We start from the equation for the electron Lorentz factor $\Gamma(z)$ (Landau \& Lifshitz 1994).

$d \Gamma(z) / d z=e E(z) / m c^{2}$,

where $z$ is the altitude of the electron above the star surface. The equation describes the change of electron energy in a nonuniform electric field $E(z)=E_{z}(z)$. (Only component that is parallel to the strong magnetic field $\mathbf{B}$ directed on $\mathbf{z}$ axes is essential). Energy losses (neither by curvature radiation nor by inverse Compton scattering) are insignificant for relevant for us values of Lorentz-factors $\Gamma$ of order of some units. We also ignore, within the polar cap, the deviation of the magnetic field lines from orthogonality to the surface of the pulsar.

The low-frequency radiation occurs at the small altitudes when the accelerating electric field $E(z)$ rises from zero level on the surface of the pulsar $z=0$. The vanishing of the electric field on the star surface is related to a small electron work function (Jones 1986) of the surface. At altitudes $z<<h$, where $h$ is the height of the gap, the field

$$
E(z)=E_{0} z / h
$$

increases linearly Arons 1981; Harding \& Muslimov 1998; Dyks \& Rudak 2000; Beskin 2010) with the altitude $z$. The velocity $V$ and the acceleration $w \equiv \ddot{z}$, expressed in terms of Lorentz factor $\Gamma$, are equal to $V=c \sqrt{\Gamma^{2}-1} / \Gamma$, and

$$
w(z)=e E(z) / m \Gamma^{3}=c^{2} \Gamma^{-3} \Gamma^{\prime}
$$

where $\Gamma^{\prime} \equiv d \Gamma / d z$. In a linear field we have

$$
\Gamma(z)=\Gamma_{0}+a z^{2}, a=e E_{0} / 2 m c^{2} h .
$$

The acceleration increases from zero when $z=0$, passes through a maximum (Fig.1) when

$$
\Gamma_{m}=6 / 5, V_{m}=c \sqrt{11} / 6
$$

(not dependent on the field) and

$$
z_{m}^{2}=2 m c^{2} h / 5 e E_{0}
$$

(for $\Gamma_{0}=1$ ) and tends to zero at approaching of electrons to relativistic velocities. The value of particle acceleration at the maximum is

$$
w_{m}=(5 / 6)^{3} c \sqrt{2 e E_{0} / 5 m h} .
$$


The height of the gap falls from these relations when the initial velocity $V(0)=V_{T} \neq 0$ and therefor $\Gamma_{0} \approx$ used henceforward the field of the form (Muslimov \& Tsygan $+V_{T}^{2} / 2 c^{2}$. Here $V_{T}<<c$ is the thermal velocity 1992)

$$
E_{0} \sim \Omega \cdot B h / c
$$

Physically, this estimate is quite obvious since on the scale of the gap the characteristic velocity due to rotation is $\Omega \cdot h$. We omit here a factor of order of unity that will be partly considered below, which contains the dependence of $E_{0}$ on the position on the polar cap. We do not discuss here the influence of general relativity effects and dependence on the angle between the axis of rotation and magnetic axis of the pulsar Beskin 2010), which does not affect the estimates. (The exception is PSR B0531+21 which is close to an orthogonal rotator.) The estimate

$$
z_{m} \approx \sqrt{(P / 1 s) \cdot\left(10^{12} G / B\right)} \cdot 1 \mathrm{~cm}
$$

confirms the legality of the conditions $z<<h$ which we use, as $h \sim 10^{4} \mathrm{~cm}$ for normal pulsars.

\section{Radiation at acceleration in the gap}

For the radiation field of accelerated electrons, we proceed from the retarded Lienard-Wiechert potentials (Jackson 1962; Landau \& Lifshitz 1994). In the problem considered the particle acceleration $\mathbf{w}$ is directed along its velocity $\mathbf{V}$. Then for the Fourier component of the wave magnetic field we have (at large distances from the radiating electron)

$\mathbf{H}_{\omega}=\frac{e}{c^{2} R_{0}} e^{i k R_{0}} \int_{-\infty}^{\infty} \frac{[\mathbf{w}(t), \mathbf{n}]}{(1-\mathbf{n} \mathbf{V}(t) / c)^{2}} e^{i(\omega t-\mathbf{k r}(t))} d t$.

Here $t=t(z)=\int_{0}^{z} d z^{\prime} / V\left(z^{\prime}\right), \mathbf{r}(t)$ is the electron radius vector, $R_{0}$ is the distance from the origin on the star surface $z=0$ to the field observation point, $\mathbf{n}=\mathbf{k} / k$.

A connection of the time $t$ with the electron coordinate $z$ is given by the integral

$t(z)=\frac{1}{c} \int_{0}^{z} \frac{\Gamma\left(z^{\prime}\right) d z^{\prime}}{\sqrt{\Gamma^{2}\left(z^{\prime}\right)-1}}$

where $\Gamma(z)$ is governed by (1).

To eliminate the logarithmic divergence at zero one should take into account in the difference $\Gamma-1$ that

of electrons on the surface of the pulsar polar cap. It is convenient to represent the Fourier component $\mathbf{H}_{\omega}$, which determines the emission spectrum, as an integral over coordinate:

$$
\mathbf{H}_{\omega}=\frac{e}{c^{2} R_{0}} e^{i k R_{0}}[\mathbf{l}, \mathbf{n}] L_{\omega}
$$

where $\quad \mathbf{l}=\mathbf{V} / V, \mathbf{n}=\mathbf{k} / k, \mathbf{l} \cdot \mathbf{n}=\cos \theta$ and

$$
L_{\omega}=\int_{0}^{h} \frac{w(z)}{V(z)\left(1-\frac{V(z)}{c} \cos \theta\right)^{2}} e^{i \omega\left(t(z)-\frac{z}{c} \cos \theta\right)} d z \text {. }
$$

Accordingly, we have for the spectral and angular radiation intensity density $d \varepsilon_{n \omega} / d \omega d o$ interesting us (Jackson 1962; Landau \& Lifshitz 1994)

$d \varepsilon_{n \omega}=\frac{e^{2}}{4 \pi^{2} c^{3}} \sin ^{2} \theta \cdot\left|L_{\omega}\right|^{2} d \omega d o$

Here $d \omega$ is the interval of frequencies, do is the interval of solid angles. It is seen from the integral $L_{\omega}$ that due to rapid oscillations the field decreases exponentially for $\omega>\omega_{c f}$, where the cutoff frequency is $\omega_{c f} \approx \pi \sqrt{2 e E_{0} / m h}$ (Fig.2). This estimate can be obtained numerically from Eq.(5) and independently from the physical consideration, supposing that the electron movement becomes relativistic: $e \int_{0}^{z_{c f}} E(z) d z=m c^{2}$ and $\omega_{c f}=2 \pi c / z_{c f}$. We obtain $\Gamma_{c f}=2, V_{c f}=\sqrt{3} c / 2$, $z_{c f}^{2}=2 m c^{2} h / e E_{0}=5 z_{m}^{2}$. The coefficient $a=1 / \lambda_{c f}^{2}$, where $\lambda_{c f}$ is the wavelength corresponding to the cutoff.

To move to the average spectra we take into account the dependence of the field from its position on the polar cap ( cf. (Dyks \& Rudak 2000)) of the form:

$$
E_{0}(r)=E_{\max }\left(1-\frac{r^{2}}{R_{P C}^{2}}\right), \quad R_{P C} \approx R \sqrt{\frac{\Omega R}{c}}
$$

( $R$ is the star radius). Assuming for the maximum field at the center of the polar cap $E_{\max } / B=\Omega \cdot h / c$, we obtain the value of the cutoff frequency $\omega_{c f}(0)=$ $\pi \sqrt{2 e \Omega B / m c}$, which is independent of the height gap. The cutoff frequency dependence on the pulsar magnetic field is shown in Fig.3.

At this frequency, the emission spectrum for the discussed mechanism must cutoff and be replaced by the next highest, forming a kink. The cutoff frequencies for a sample of normal pulsars had been found in 


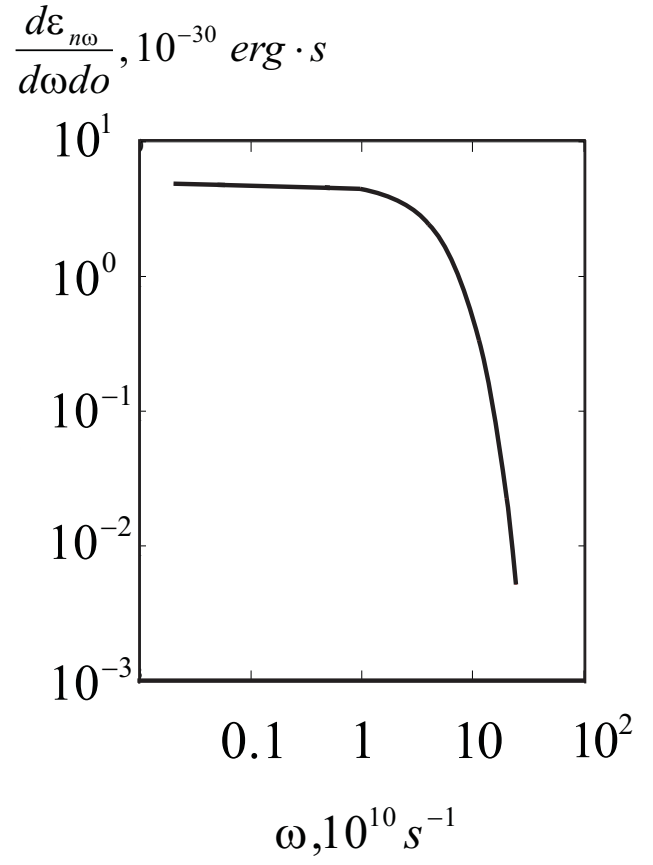

Fig. 2 The emission spectrum of a particle at acceleration in the electric field linearly increasing with altitude $\mathrm{z}$ above the surface of the star (the numerical calculation of (5) for $\left.\theta=\pi / 8, B=10^{12} G, P=1 s\right)$

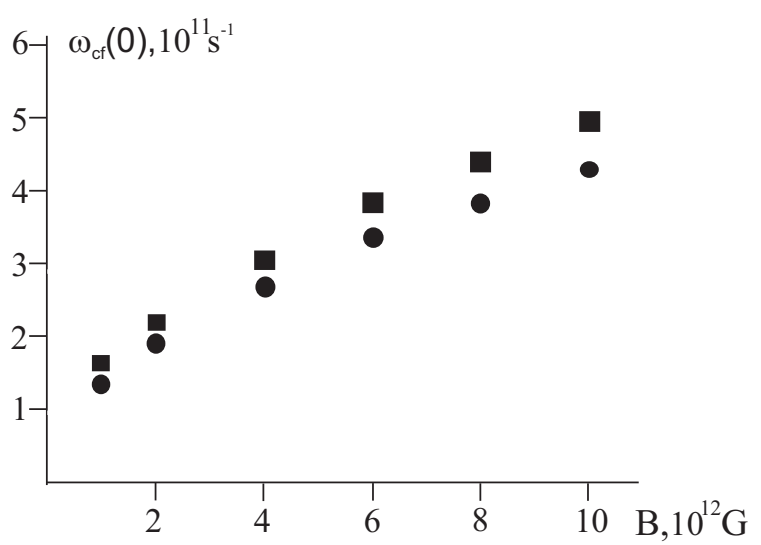

Fig. 3 The dependence of cutoff frequency on the magnetic field for $\theta=\pi / 8, P=0,07 s$. Squares correspond to $\omega_{c f}(0)$, circles - to numerical integration of $\mathrm{E}_{\omega}$
(Malofeev \& Malov 1980). The dependence on the period for $B=2 \cdot 10^{12} G$ coincides for $\hat{\nu}_{c f}=V_{c f} / 2 \pi z_{c f}$

$\hat{\nu}_{c f} \approx \sqrt{2} \cdot 10^{9} H z \sqrt{\left(\frac{B}{2 \cdot 10^{12} G}\right) \cdot\left(\frac{1 s}{P}\right)}$,

(this theory)

$$
\tilde{\nu}_{c f} \approx 1.4 \cdot 10^{9} \mathrm{~Hz}\left(\frac{1 s}{P}\right)^{0.46 \pm 0.18}
$$$$
\text { (Malofeev \& Malov 1980). }
$$

(The tilde over frequency means the experimental value). The dependence $\hat{\nu}_{c f}$ on $B$ and other parameters, (see as example (Dyks \& Rudak 2000)) determining the electric field in the gap, is available for testing. It can also serve as a criterion for the correctness of this description. Below we use the cutoff frequency $\nu_{c f}=c / z_{c f}$ corresponding decrease of intensity radiation of a single electron to $e$ times (see Fig.2). The actual frequency of the cutoff may be less than the estimated cutoff frequency both due to the fact that the actual accelerating field in the gap is less than the adopted estimates and the cutoff corresponds to higher values of gamma-factor.

\section{Average spectra}

Now we consider the emission spectrum of a large number of electrons accelerated in the electric field of the gap. From the intensity of a single particle (taking into account that in the electric field, linearly increasing from zero on the star surface, the acceleration maximum is proportional to the square root of $E_{0}$ )

$I(r)=\frac{2 e^{2} w^{2}(r)}{3 c^{3}} \approx \frac{e^{3} E_{0}(r)}{m c h}$,

and changing the spectrum in the Fig. 2 by the stepfunction, we turn to the spectral density for $\omega<\omega_{c f}(r)$ :

$I(r, \omega) d \omega \approx I(r) \frac{d \omega}{\omega_{c f}(r)}$,

where $\quad \omega_{c f}(r)=\pi \sqrt{\frac{2 e E_{0}(r)}{m h}}$.

Accordingly, the frequency range of radiation of the single particle has the form

$\omega^{2} \leq \omega_{c f}^{2}(r)=\frac{2 \pi^{2} e E_{\max }}{m h}\left(1-\frac{r^{2}}{R_{P C}^{2}}\right)$, 
therefore for the spectrum (in an incoherent case) we obtain

$$
\begin{gathered}
I(\omega) \propto 2 \pi \int_{0}^{b(\omega)} r d r I(r, \omega) N, \\
b(\omega)=R_{P C} \sqrt{1-\frac{\omega^{2}}{\omega_{c f}^{2}(0)}},
\end{gathered}
$$

where $N$ is the number of emitting particles measured through the current across the polar cap. Integration over the polar cap gives

$$
\begin{array}{r}
I(\omega) \propto \int_{0}^{b(\omega)} r d r \sqrt{1-\frac{r^{2}}{R_{P C}^{2}}} \propto 1-\frac{\omega^{3}}{\omega_{c f}^{3}(0)}, \\
\omega_{c f}(0) \approx \pi \sqrt{\frac{2 e E_{\max }}{m h}}, \omega \leq \omega_{c f}(0),
\end{array}
$$

i.e. the spectrum is flat and has a cut off at the frequency $\omega_{c f}(0)$.

Let us now consider the coherence of the emission Ginzburg et al. 1969; Ginzburg 1971; Kuzmin \& Solov'ev 1986; Golgreich \& Keeley 1971). In the discussed mechanism only thin a disk with $z<z_{c f}$ emits and the disk thickness is less then any wavelengths emitted. Therefore, in our case there is no need to raise additional assumptions about formation in the longitudinal direction coherently emitting electron bunches, which represents up to now a significant difficulty in all theories of pulsar radio emission. However, we must assume that in the disk plane a fragmentation takes place to coherently emitting regions, just as it is supposed in explaining the drift of subpulses (Lyne \& Graham-Smith 1998). Here we restrict ourselves to the case when fragmentation occurs in a region with a transverse dimension

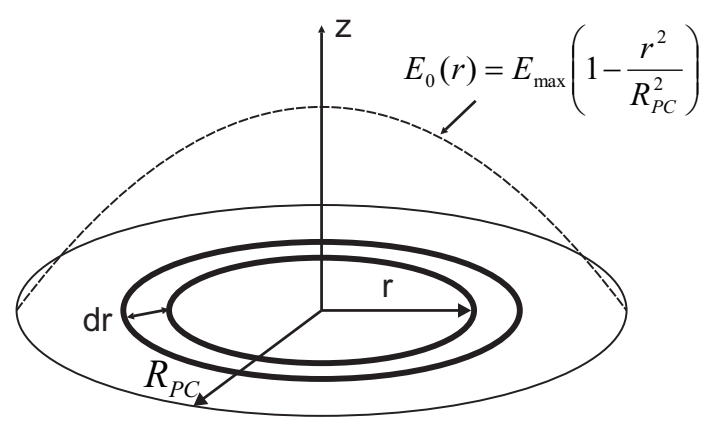

Fig. 4 Formation of average spectrum of radio emission. The dotted line shows the change of the longitudinal electric field with the distance from the magnetic axis to the boundary of pulsar polar cap less than the length of the radiated wave3. In this case, we find the upper limit of radiated power at a given fragmentation. For this the total number of particles $N$ is divided into coherent blocks (which are different for different wavelengths $\lambda$ and depend on blocks position)

$N=N_{\text {block }} \cdot \frac{2 \pi}{\Sigma_{P C}} \int_{0}^{R_{P C}} r d r N_{c o h}(r)$.

Inside of the blocks the intensity is proportional to the square of the particle number $N_{c o h}(r)$. The required limitation (Golgreich \& Keeley 1971) of block height $\bar{z}$ is provided by the condition $\lambda>\bar{z} \approx z_{c f}$, where $z_{c f}$ is the height to which the radiation mechanism acts. The blocks are summed additively

$I(\omega) \propto 2 \pi \int_{0}^{b(\omega)} r d r I(r, \omega) N_{\text {block }}(r) N_{c o h}^{2}(r)$.

The condition $\lambda>\bar{z}$ for all emitted waves is fulfilled automatically in our case that is very important for realization a coherent emission mechanism. As for division of the polar cap on coherent areas, at this stage we must restrict the discussion only by assumptions. Dividing polar cap on the regions of order lambda, we obtain, in coherent case, the lower estimates for intensity of radiation due to the minimum square of the number of particles in each region.

Obviously, the number of blocks equals to $N_{\text {block }} \approx$ $\left(R_{P C} / \lambda\right)^{2}$, and the number of particles in the block is $N_{\text {coh }} \approx \pi \lambda^{2} \bar{n}_{e} \bar{z}$. Then for radiation spectrum we have

$I(\omega) \propto 2 \pi \int_{0}^{b(\omega)} r d r \frac{1}{\lambda^{2}} \sqrt{E_{0}(r)} \lambda^{4} \bar{z}^{2} \propto$

$\propto \frac{1}{\omega^{2}} \int_{0}^{b(\omega)} \frac{r d r}{\sqrt{1-r^{2} / R_{P C}^{2}}}=\frac{1}{\omega^{2}}\left(1-\frac{\omega}{\omega_{c f}(0)}\right)$.

where $b(\omega)$ is given by Eq. (11) and the frequency $\omega_{c f}(0)$ is determined accordingly to Eq. (12). We have obtained a power-law spectrum with spectral index 2

${ }^{3}$ In the case of a thin disk it is possible, in principle, a coherent radiation of considerably larger in transverse dimension regions, bounded by the scale $\sqrt{R_{0} \lambda}$ (Smith 1977), which gives for the upper boundary of power a significantly large value. When $R_{0}=$ $R_{P C}$ it gives about a dozen of coherently emitting regions on the polar cap, comparable with the number of observed ones in the drift of subpulses Deshpande \& Rankin 1999; Smith 1977; Palfrevman et al. 2011). See also (Young 2003). 
(Fig.5), which is typical for the majority of pulsars. Let us note, that Eq.(12) and (15) are only the asymptotics of the exact spectra. In reality there is a low frequency break (or turnover) at the frequency $\omega_{t r} \approx 0.1 \omega_{c f}$ (Malov 2004). Such spectrum behaviour can be obtained in the discussed mode 4 and we have considered in detail the physical meaning of that break and its position in the separate paper (Kontorovich. Flanchik 2013). Near the cutoff frequency the emission spectrum can not be considered strictly as a power law. For a small number of measured values it may be perceived as a kink. The spectral index depends on transverse dimensions of coherent blocks, which it seems also reveals themselves in the geometry of the subpulse drift.

The coherence provides a reasonable estimate of the intensity of radio emission. The rough estimate of the power of radio emission has the form

$I_{R} \sim N_{\text {block }} N_{c o h}^{2} I_{1}, \quad I_{1} \approx \frac{e^{3} E_{0}}{m c h}$

where $I_{1}$ is the radiation power of a single particle at the maximum of acceleration. Here $N_{\text {block }} \approx$ $\left(R_{P C} / \lambda_{\max }\right)^{2}$, where $\lambda_{\max } \sim 10^{2} \mathrm{~cm}$ is a wavelength corresponding to the maximum in the spectrum of radio emission. Writing an estimate for the number of particles in a coherent block in the form $N_{\text {coh }} \approx$ $\pi \lambda_{\text {max }}^{2} \bar{z} \bar{n}_{e}$, where $\bar{n}_{e} \sim n_{G J}=\Omega B /(2 \pi c e)$ is the average density of particles near the surface, we have $I_{R} \sim\left(\pi R_{P C} \lambda_{\max } \bar{z} n_{G J}\right)^{2} I_{1}$, whence the estimate $I_{R} \sim$ $\lambda_{\max }^{2} \Omega^{3} R^{3} B^{2} / c^{2}$ results. For the parameters $B=$ $4 \cdot 10^{12} \mathrm{G}, P=1 \mathrm{~s}$ it leads to $I_{R} \approx 10^{28} \mathrm{erg} / \mathrm{s}$, which agrees well with the data on the radio luminosity of pulsars. For the fast rotating pulsar B0531+21 with high

${ }^{4}$ Note, that there is a dynamical but not a dissipative reason (cf. Ochelkov \& Usov 1980)) for a turnover.

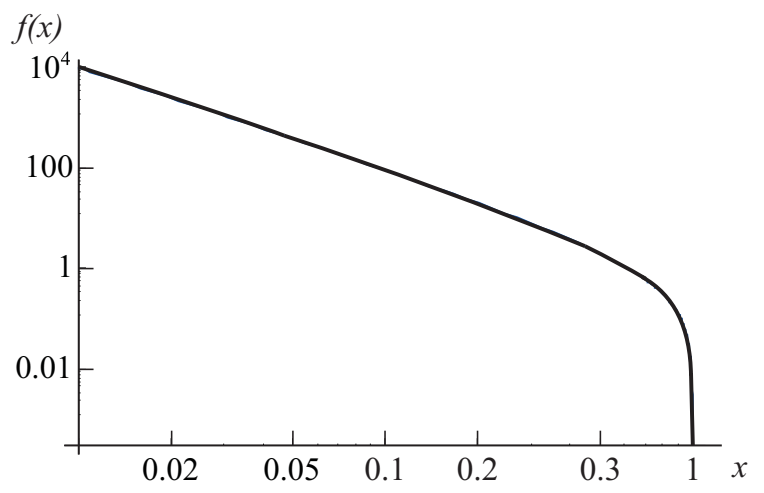

Fig. 5 Function $f(x)=(1-x) / x^{2}, x=\omega / \omega_{c f}(0)$ that defines the spectrum of coherent emission of electrons accelerated in the vacuum gap magnetic field this estimate reaches $10^{32} \mathrm{erg} / \mathrm{s}$ taking into account its proximity to the orthogonal rotator.

Note, that the assumption about change of radiation mechanisms in the high frequency cutoff region allows in principle to explain (Kontorovich \& Flanchik 2011) the main pulse disappearance of PSR B0531+21 at frequencies near $8 \mathrm{GHz}$ (Hankins \& Eilek 2007). Really, from our point of view at lower frequencies the radiation at longitudinal acceleration of subrelativistic electrons gives the principal contribution to the main pulse. This emission vanishes at the cutoff frequency that may lead to disappearance of the pulse. The radiation due to low-frequency tail of narrow directed aberrational relativistic mechanism remains. This anisotropic radiation does not fall to the main pulse window but reveals itself in the interpulse if its line of sight is more close to the magnetic axis.

Note also that the discussed mechanism of radio emission (for which the place of radiation is definite and occupies a thin layer near the surface of the star) may use for checking the new methods to determine the generation location by a dispersion delay of the signal in the magnetospere (Hassall, et al. 2012). The accuracy of such methods is limited now by the lack of the adequate knowledge of magnetosphere properties.

\section{Conclusions}

We have found above that the radiation of accelerating electrons in the electric field, slow increasing from zero on the star surface, entirely comes to the radio spectral band. This is the main result of the work.

The considered approach allows us explain the radio emission for the pulsars from Pushchino sample, including the position of the spectrum cutoff. As a result, we obtain a power-law spectrum, which arise at averaging over the polar cap due to dependence of the accelerating electric field on its position. The density radiation in the gap is large without any assumptions about the gap as a cavity (cf. (Kontorovich 2009)). It explains also the part of the gamma-ray emission from pulsars and its correlation (Bilous, et al. 2011) with giant pulses (Kontorovich \& Flanchik 2008).

The radiation with the linear acceleration was considered in (Melrose et al. 2009a, b) for acceleration in a so strong electric field on the star surface, when an electron reaches relativistic velocities in a time shorter then the period of the wave emitted. In this case the considered effects are absent.

The obtained results make it also possible to compare the theories of accelerating fields in the inner gap (Harding \& Muslimov 1998; Dyks \& Rudak 2000) 
(that is the foundation of all physics of pulsars) with observations, transforming them from a "thing in itself" that is not available to direct observations, in the "thing for us".

Acknowledgements We are grateful to our colleagues from RI NAS of Ukraine and to participants of PRAO-2011, JENAM-2011 and NS-2011 conferences for discussions, to M. Azbel', O. Ulyanov and V. Usov for useful comments and to N. Kisilova, Y. Schukin and V. Tsvetkova for their help in translation of the text. The authors are extremely grateful to reviewer for a kindly and qualified critique of this work. 


\section{References}

Arons, J.: Pair creation above pulsar polar caps - Steady flow in the surface acceleration zone and polar CAP X-ray emission. Astrophys. J. 248, 1099 (1981)

Beskin, V.S.: MHD Flows in Compact Astrophysical Objects. Springer, 425 p. (2010)

Beskin V.S., Gurevich A.G. \& Istomin Ya.N.: Physics of the pulsar Magnetosphere. Cambridge University Press, Cambridge (1993)

Beskin, V.S. \& Philippov A.A.: On the mean profiles of radio pulsars I: Theory of the propagation effects Mon. Not. R. Astron. Soc. 425, 814 (2012); arXiv:1107.3775 v2

Bilous, A.V., Kondratiev,V.I., McLaughlin, M.A., et al: Correlation of Fermi photons with high-frequency radio giant pulses from the Crab pulsar. Astrophys. J. 728, 110 (2011); arXiv: 1101.1434

Camilo, F.; Ransom, S. M.; Chatterjee, S.; Johnston, S.; Demorest,P.: PSR J1841-0500: A Radio Pulsar That Mostly is Not There. Astrophys. J. 746, article id.63 (2012); arXiv: 1111.5870

Deshpande, A., Rankin, J.: Pulsar Magnetospheric Emission Mapping: Images and Implications of Polar CAP Weather. Astrophys. J. 524, 1008 (1999)

Dyks, J., Rudak, B.: Approximate expressions for polar gap electric fields of pulsars. Astron. Astrophys. 362, 1004 (2000); ArXiv: astro-ph/0006256

Gil, J., Melikidze, G.I: Angular beaming and giant subpulses in the Crab pulsar. Astron. Astrophys. 432, L61 (2005)

Ginzburg, V.L.: Pulsars Present State of the Problem. Physics-Uspekhi 14, 229 (1971)

Ginzburg, V.L., Zheleznyakov, V.V., Zaitsev, V.V.: Coherent mechanisms of radio emission and magnetic models of pulsars. Physics-Uspekhi 12, 378 (1969)

Golgreich, P., Keeley, D.: Coherent Synchrotron Radiation. Astrophys. J. 170, 463 (1971)

Hankins, T.H., Eilek, J.A.: Radio emission signatures in the Crab pulsar. Astrophys. J. 670, 693 (2007); arXiv:0708.2505

Harding, A.K., Muslimov, A.G.: Particle acceleration zones above pulsar polar caps: electron and positron pair formation fronts. Astrophys. J. 508, 328 (1998)

Hassall,T. E. , et al: Wide-band Simultaneous Observations of Pulsars: Disentangling Dispersion Measure and Profile Variations Astron. Astrophys. 543, A66 (2012)

Izvekova V.A., Kuzmin A.D., Malofeev V.M. et al.: Radio spectra of pulsars Astrophys. Space Sci. 78, 45 (1981)

Jackson, J.D.: Classical electrodynamics. Wiley, NY, 1962

Jones, P.B.: Properties of condensed matter in very strong magnetic fields. Mon. Not. R. Astron. Soc. 218, 477 (1986)

Kazbegi A. Z., Machabeli G. Z., Melikidze G. I.: article title On a possible mechanism of pulsar radiation, in Proc. IAU Collog. 128, The Magnetospheric Structure and Emission Mechanisms of Radio Pulsars, ed. T.H. Hankins, J.M. Rankin \& J.A. Gil (Zielona Gora: Pedagogical Univ. Press) 232 (1992)

Kontorovich, V.M.: Electromagnetic tornado in the vacuum gap of a pulsar, Journal of Experimental and Theoretical Physics. JETP 110, 966 (2010)
Kontorovich, V.M.: On high brightness temperature of pulsar giant pulses. ArXiv: 0909.1018 (2009)

Kontorovich, V.M., Flanchik, A.B.: On the connection between the gamma and radio spectra of pulsars. JETP $\mathbf{1 0 6}$, 869 (2008)

Kontorovich, V.M., Flanchik, A.B.: High-frequency cutoff of spectrum and change in the mechanism of radio emission in pulsars. Actual Problems of Extragalactic Astronomy, Pushino, Russia, 19 - 21 April 2011. http://www.prao.ru/conf/28_conf/registration/docs.php

Kontorovich, V.M., Flanchik, A.B.: Radio emission with acceleration of electrons in a polar gap of a pulsar. Physics of Neutron Stars - 2011, Saint-Petersburg, Russia, 11 - 15 July 2011, Abstract book, p.75.

http://www.ioffe.ru/astro/NS2011/index.html

Kontorovich, V.M., Flanchik, A.B.: Correlation of pulsar radio emission spectrum with peculiarities of electron acceleration in polar gap. JETP 143, 1 (2013); astro$\mathrm{ph} / 1210.2858$

Kramer, M., Lyne, A. G., OBrien, J. T., Jordan, C. A., Lorimer, D. R.: On the nature of the intermittent pulsar PSRB1931+24 science 312, 549 (2006)

Kuzmin, A.D., Solov'ev, A.G.: Model pulsar radio spectra and pulse profiles. Soviet Astron. 63, 62 (1986)

Lane, A.G.,Burgay, M., Kramer, M., et al.: A double-pulsar system. Science 303, 1153 (2004)

Landau, L.D., Lifshitz, E.M.: The Classical Theory of Fields. Oxford, Butterworth-Heinemann (1994)

Lorimer, D.R., Lyne, A.G., McLaughlin, M.A. , Kramer, M., Pavlov, G.G., \& Chang, C.: Radio and X-ray observations of the intermittent pulsar J1832+0029 Astrophys. J. 758, 141 (2012)

Lyne, A.G., Graham-Smith, F.: Pulsar Astronomy. Cambridge University Press, Cambirdge (1998)

Malofeev, V.M.: Pulsar Radio Spectra. In Pulsars: Problem \& Progress. ASP Confer. Series, 105, 271 (1996)

Malofeev, V.M.: Catalogue pulsar radio spectra (in Russian). PRAO ASC FIAN, Pushchino (1999)

Malofeev, V.M., Malov, I.F.: Average pulsar spectra and their interpretation. Soviet Astron. 57, 90 (1980)

Malov, I.F., Malofeev, V.M.: Radio spectra of pulsars. II The interpretation. Astrophys. Space Sci. 78, 73 (1981)

Malofeev, V.M., Malov, I.F.: The nature of high frequency cut-offs in spectra of pulsars . Soviet Astron. 68, 362 (1991)

Malov, I.F.: Radio Pulsars. Nauka, Moscow (2004)

Malov, I.F. \& Machabeli, G.Z.: Anomalous pulsars. Nauka, Moscow (2004)

Manchester, R.N.: Radio Emission Properties of Pulsars. in W.Becker, ed, Neutron Stars and Pulsars. SpringerVerlag, Berlin Heidelberg (2009)

Manchester R. \& Taylor J.: Pulsars. Freeman, San Francisco (1977)

Maron O., et al.: Astron. Astrophys. Suppl. Ser. 147, 195 (2000)

Melrose, D.B., Rafat, M.Z., Luo, Q.: Linear Acceleration Emission. I. Motion in a Large-Amplitude Electrostatic Wave. Astrophys. J. 698, 115 (2009)

Melrose, D.B., Luo, Q.: Linear Acceleration Emission. II. Power Spectrum. Astrophys. J. 698, 124 (2009) 
Moffett, D., Hankins, T.: Multifrequency radio observations of the Crab pulsar. Astrophys. J. 468, 779 (1994); ArXiv: astro-ph/9604163.

Muslimov, A.G., Tsygan, A.I.: General relativistic electric potential drops above pulsar polar caps. Mon. Not. R. Astron. Soc. 255, 61 (1992)

Ochelkov, Yu.P., Usov, V.V.: The nature of low-frequency out-off in the radio emission spectra of pulsars Nature 309, 332 (1984)

Palfreyman, J.L., et al: Consecutive Bright Pulses in the Vela Pulsar. Astrophys. J. 735, L17 (2011); arXiv:1106.4625

Ruderman, M.A., Sutherland, P.G.: Theory of pulsars: polar gaps, sparks, and coherent microwave radiation. Astrophys. J. 196, 51 (1975)

Sieber, W.: Reviewing Pulsar Spectra, Proceedings of the 270. WE-Heraeus Seminar on: Neutron Stars, Pulsars and Supernova Remnants Physikzentrum Bad Honnef, Germany, Jan. 21-25, 2002, eds. W. Becker, H.Lesch \& J. Tr.umper, MPE Report 278, pp. 171- 176; astro-ph/0208571 1

Smith, F.G.: Pulsars. Cambridge Univ. Press, Cambirdge

Smits, J.M. et al.: The geometry of PSR B0031-07. Astron. Astrophys. 465, 575 (2007)

Sturrock, P.A.: A model of pulsars. Astrophys. J. 164, 529 (1971)

Usov, V.V.: On two-stream instability in pulsar magnetospheres. Astrophys. J. 320, 333 (1987)

Young M.D.T.: A Resonant-Mode Model of Pulsar Radio Emission. in IAU Symp. 218, Young Neutron Stars and Their Environments, eds. F. Camilo and B.M. Gaensler (San Francisco: ASP, 2004), p.365; astro-ph/0310411 